\title{
Risperidone, quetiapine, and olanzapine adjunctive treatments in major depression with psychotic features: a comparative study
}

This article was published in the following Dove Press journal:

Neuropsychiatric Disease and Treatment

II April 2013

Number of times this article has been viewed

\section{A Gabriel \\ Departments of Psychiatry and Community Health Sciences, University of Calgary, Calgary, $A B$, Canada}

Objectives: The purpose of this study was to compare the effectiveness of novel antipsychotics in the treatment of psychotic depression.

Method: Consecutive patients who were admitted $(\mathrm{n}=51)$ with a confirmed diagnosis of major depression with psychotic features (delusions or hallucinations or both) participated in this open-label, naturalistic study. All patients were treated with selective serotonin reuptake inhibitors (SSRIs) and serotonin-norepinephrine reuptake inhibitors (SNRIs) (citalopram or venlafaxine extended release [XR]), and atypical antipsychotic agents were added, as tolerated, during the first week of initiating the citalopram or venlafaxine. There were patients $(n=16)$ who received risperidone, who received quetiapine $(n=20)$, and who received olanzapine $(n=15)$, as an adjunctive treatment to either citalopram or venlafaxine for at least 8 weeks. Outcome measures included the Clinical Global Impression-Severity subscale (CGI-S), as the primary outcome measure, as well as the Hamilton Rating Scale for Depression-21 item (HAM-D21) and the Brief Psychiatric Rating Scale (BPRS). Tolerance to treatments and weight changes were monitored over the period of the trial.

Results: All patients completed the trial with no drop outs. At 8 weeks, there was a statistically significant $(P<0.001)$ clinical improvement in all outcome measures for both the depressive and psychotic symptoms, for all three groups of atypical adjunctive treatments. Utilizing analysis of variance (ANOVA), there were no significant differences between the three adjunctive treatment groups in outcome measures. The three antipsychotic agents were equally tolerated. At 8 weeks there was slight increase in weight in the three treatment groups, which was statistically significant $(P>.01)$ in the olanzapine group.

Conclusion: Quetiapine, risperidone, and olanzapine, given as adjunctive treatment with SSRIS or SNRIs can significantly and equally improve depressive and psychotic symptoms, in the short-term treatment of major depression with psychotic features. The author recommends that large controlled trials be conducted to examine the differences in long-term efficacy and tolerance between the atypical antipsychotic agents, in the treatment of major depression with or without psychotic features.

Keywords: depression, novels, antipsychotics, treatment, augmentation

\section{Introduction}

Major depressive episodes with psychotic features are relatively frequent in the general population, affecting four in 1000 individuals, and it was demonstrated that approximately $15 \%-20 \%$ of the cases that fulfill the criteria for major depressive episode are of the psychotic subtype. ${ }^{1}$ However, psychotic depression has not been studied to the same extent as other mental disorders with similar prevalence and remains an underdiagnosed and probably undertreated mental disorder. ${ }^{2}$ 
One thorough review focused on recognizing the features of psychotic depression, the success of current treatment options, and the new treatments under investigation. ${ }^{3}$ Major depression with psychotic features, while fairly common, is frequently misdiagnosed. The symptoms seen in these patients are those of an overall severe depressive disorder, with psychomotor impairment (retardation or agitation), guilt, suicidal preoccupation, and neuropsychological impairment. A number of biological characteristics and behavioral symptoms are specific to patients suffering from psychotic depression and differ significantly from those of nonpsychotic depression. Psychotic depression is seen in patients of all ages, has a high short-term morbidity, and risk of suicide. Fortunately, there has been strong evidence and data to support the use of antipsychotics in combination with antidepressants for major depression with psychotic features. ${ }^{3}$ If patients with psychotic depression were treated appropriately, these risks for morbidity and mortality could be reduced.

Traditionally, psychotic depression was treated with antidepressants augmented with the typical antipsychotic agents. These drugs were, to large extent, found to be associated with a high risk for excessive extrapyramidal side effects. However, the atypical antipsychotic agents, such as risperidone, quetiapine, and olanzapine, have been shown to have a lower risk of the same. ${ }^{4,5}$

Weizman et al (2001) reviewed the utilization of atypical antipsychotics in mood disorders and pointed out that atypical antipsychotics can serve as adjunctive as well as alternative agents in the treatment of drug-resistant mood disorders. Olanzapine and risperidone add-on treatment were found to be effective for major depression with psychotic features, and good results were achieved with currently available atypical antipsychotics (clozapine, risperidone, olanzapine, quetiapine, and ziprasidone) in reducing the symptoms of acute mania, especially when added to mood stabilizers. The role of atypical antipsychotics in maintenance and prophylactic treatment is not yet clear. Although there are differences in the side-effect profiles of the various atypical antipsychotics, their long-term use is limited by adverse effects, such as extrapyramidal symptoms, weight gain, somnolence, and sexual dysfunction. ${ }^{6}$

An earlier review ${ }^{7}$ concluded that placebo-controlled studies have shown that second-generation antipsychotics have antidepressive effects that are significantly stronger than those of the traditional neuroleptics. In addition, it has been demonstrated that this antidepressive effect can only partially be explained as a secondary effect of the improvement of positive and negative symptoms and is, conversely, apparently predominantly due to a direct (primary) effect on depressive symptoms. It is of special relevance in this context that the antidepressive effect of second-generation antipsychotics was recently demonstrated in depression.

In another recent review, ${ }^{8}$ examining the guidelines for psychotic depression treatment, the authors found that six out of nine national and international guidelines for psychotic depression suggested the combination of antidepressants and antipsychotics for first-line treatment.

There is growing evidence to support the efficacy of the combination of antidepressants and antipsychotics in the treatment of depressed patients with or without psychotic depression. For example, recently Maneeton et al, ${ }^{9}$ in a metaanalysis, demonstrated that based on the available evidence obtained from three randomized, controlled trials (RCTs), quetiapine XR was effective for adult patients with major depressive disorder. The high dropout rate, due to adverse events, suggested that some major depressive disorder patients may not be able to tolerate quetiapine XR., ${ }^{9,10}$

In a number of multicentre, randomized, double-blind, placebo-controlled studies, the quetiapine fumarate XR demonstrated efficacy as monotherapy or as an adjunctive therapy in the treatment of partially responsive depression and in the management of bipolar depression. ${ }^{11-13}$ Atypical antipsychotics have also demonstrated efficacy in relieving comorbid anxiety in patients with mood disorders. For example, quetiapine fumarate $\mathrm{XR}$, as adjunctive therapy to selective serotonin reuptake inhibitors (SSRIs) or as monotherapy, has been claimed to be especially more useful in patients with high levels of anxiety and in maintaining overall functioning and sleep quality than placebo, in patients with major depressive disorder. ${ }^{14-16}$

There is limited published research that has examined direct comparisons between different antipsychotics in patients with psychotic depression. However, there is a large-scale, open-label study ( $\mathrm{n}=554$ patients) comparing the efficacy of risperidone and quetiapine in psychotic depression. The authors indicated that both drugs reduced the mean score on the Hamilton Rating Scale for Depression-21 item (HAMD-21). In this study, quetiapine demonstrated superiority over risperidone in producing greater improvement of both psychotic and depressive symptoms. ${ }^{17}$

The purpose of the present study was to test the hypothesis that quetiapine, risperidone, and olanzapine used as adjunctive therapy to SSRIs or serotonin-norepinephrine reuptake inhibitors (SNRIs) will improve depressive and psychotic 
symptoms, in the short-term treatment of major depression with psychotic features.

This was the first direct comparison study of the efficacy and tolerability of the adjunctive treatment of the three atypical antipsychotics, risperidone, quetiapine, and olanzapine, in the treatment of major depression with psychotic features.

\section{Materials and methods}

The study was carried out at the Peter-Lougheed Hospital, involving consecutively admitted from either the inpatient or outpatient units with the diagnosis of major depression with psychotic features, who were treated with SSRI or SNRI antidepressants in conjunction with quetiapine, olanzapine, or risperidone, in the absence of other antipsychotic medication. The proposal for the study was granted approval from the conjoint scientific and ethics committee of the University of Calgary.

\section{Study design}

This was an 8-week open-label, naturalistic study of add on risperidone quetiapine, or olanzapine for 8 weeks at least, as an adjunctive treatment to SSRIs or SNRIs, in patients with the confirmed diagnosis of major depression with psychotic features. All patients fulfilled the diagnostic criteria for DSMIV diagnosis ${ }^{18}$ of major depression with psychotic features. Diagnoses were confirmed using the Mini-International Neuropsychiatric Interview. ${ }^{19}$

\section{Patient population}

Patients in the study included (1) both males and females, 18-65 years of age, and (2) only patients with confirmed diagnosis of psychotic depression, who were treated with SSRI or SNRI antidepressants and the atypical antipsychotics risperidone, quetiapine, or olanzapine, as an adjunctive treatment.

Patients who were excluded from the study were those who (1) had a diagnosis of major medical disorders or alcohol and drug abuse, (2) all patients with the diagnosis of schizophrenia, delusional disorders, schizoaffective disorder, or other psychotic disorder during the index episode, (3) patients with mental handicap, and (4) acutely suicidal patients.

\section{Drug administration}

The SSRI or SNRI antidepressants (citalopram or venlafaxine $\mathrm{XR}$ ) were initiated, and the dose was titrated gradually against patients' tolerance, to a maximum of citalopram $60 \mathrm{mg}$ a day or venlafaxine XR $300 \mathrm{mg}$ a day. The atypical antipsychotics (risperidone, quetiapine, and olanzapine) were added to the existing SSRI or SNRI antidepressants during the first week of initiating the antidepressants. The dose of the novel agents was titrated at weekly intervals, as tolerated, with $0.25-0.5 \mathrm{mg}$ increments of risperidone, to a maximum of $2 \mathrm{mg}$ a day; $25-50 \mathrm{mg}$ increments of quetiapine, to a maximum dose of $300 \mathrm{mg}$ a day; and $2.5-5 \mathrm{mg}$ increments for olanzapine, to a maximum of $15 \mathrm{mg}$ a day. All novels were prescribed as a single dose at bedtime. No patients received other concomitant antipsychotics during the 8 weeks of the adjunctive treatments with any of the three atypical antipsychotics. Given the fact that the study was an open naturalistic study, the decision regarding the type of the atypical antipsychotic selected was based on the clinical decision of the treating physician, in consultation with the treating team.

\section{Outcome measures}

The data that was collected included the Clinical Global Impression-Severity subscale (CGI-S) as the primary outcome measure, as well as the Global Improvement subscale, ${ }^{20}$ the HAM D-21, ${ }^{21}$ and the Brief Psychiatric Rating Scale (BPRS). ${ }^{22}$ Systematic reporting of psychotic symptoms and abnormal involuntary movements (extrapyramidal symptoms), before and after treatments with the novel antipsychotics, and weight changes over 8 weeks were also recorded. Patients' demographic and clinical data, including reporting of side effects, that had been collected, during regular patient visits to the hospital or the clinician's office, were collected, summarized and tabulated.

\section{Data analysis}

Descriptive statistics was used to summarize the demographic variables, and the frequencies of psychotic symptoms, suicidal ideas, suicidal behavior, and medication dose for each atypical antipsychotic. To examine the changes in the CGI-S, in HAMD-21, the BPRS, and in weight over time, we employed the paired $t$-test, by pairing scores at baseline, before the addition of the atypical antipsychotics, and those at 8 weeks. The one-way analysis of variance (ANOVA) was utilized to examine differences in the outcome measures scores between the three adjunctive treatment groups. The dependent variables were the CGI-S, HAMD-21, BPRS, and weight, at baseline and at 8 weeks of treatment. The independent variables were the three adjunctive medication groups.

\section{Results}

In all, 51 patients completed at least an 8-week trial of one of three atypical antipsychotic agents (risperidone, 
quetiapine, or olanzapine) given as adjunctive treatment with SSRI or SNRI antidepressants. Therefore, there were three SSRI- or SNRI-adjunctive treatment groups; the risperidone, the quetiapine, and the olanzapine groups.

Table1 displays the demographic characteristics, including the number of men and women in each treatment group, and the mean dosage for each novel antipsychotic. No patients were administered any other antipsychotics at the same time. The mean age distribution was 41.5 years (standard deviation [SD]: 9.4) in the risperidone group, 42 years (SD: 10.2) in the quetiapine group, and 41 years (SD: 11.6) in the olanzapine group. There was no significant difference in age between the three groups.

All patients exhibited psychotic symptoms in the form of either delusions, hallucinations, or both. Nine patients (17\%) had exhibited suicidal behavior before treatment; after adjunctive treatment, there was no reported suicidal behavior or thoughts in any of the three groups. Before treatment, 36 (71\%) patients presented with delusions and $18(35 \%)$ patients reported auditory hallucinations. The delusions were mostly described as mood congruent, such as persecutory, hypochondriacal, guilty, or nihilistic delusions. Psychotic symptoms improved significantly with treatment in the three treatment groups. At 8 weeks of the adjunctive treatment, few patients were still exhibiting psychotic symptoms. Table 1 summarizes the psychotic symptoms and the details of treatments per each novel adjunctive treatment group.

Paired $t$-tests and ANOVA were utilized to examine and compare mean changes in the outcome measures, the CGI-S, the HAMD-21, the BPRS, and weight, at baseline and at 8 weeks of the atypical augmentation.
Table 2 displays the results from paired $t$-test for the outcome measures (CGI-S, HAMD-21, BPRS), and weight changes at 8 weeks, for each treatment group. A close inspection of Table 2 shows that there was significant improvement in all outcome measures at $P<0.001$, and there were no significant weight changes at 8 weeks of the study for any of the three atypical adjunctive treatments.

On the Global Impression subscale, among the 16 patients in the risperidone group, there were three (18\%) patients who were very much improved, ten (64\%) patients who were much improved, and three (18\%) patients who were minimally improved. Among the 20 patients in the quetiapine group, there was one (5\%) patient who was very much improved, eleven (55\%) patients who were much improved, seven $(35 \%)$ patients minimally improved, and one $(5 \%)$ patient with no change; and among the 15 patients in the olanzapine adjunctive treatment group, there were two (13\%) patients who were very much improved, seven (47\%) patients who were much improved, and six (40\%) patients who were minimally improved.

Employing ANOVA, at 8 weeks, there were no significant differences between the three treatment groups for any of the outcome. Table 3 summarizes the changes and the differences in the outcome measures and weight change between the three groups.

\section{Tolerance to the adjunctive treatment}

The three novel antipsychotics, risperidone, quetiapine, and olanzapine were well tolerated, with insignificant adverse effects at 8 weeks of treatment. Among the patients in the risperidone group, there was one patient who reported extrapyramidal symptoms in the form of mild tremors and

Table I Demographic variables

\begin{tabular}{|c|c|c|c|c|}
\hline & $\begin{array}{l}\text { Risperidone } \\
(n=16)\end{array}$ & $\begin{array}{l}\text { Quetiapine } \\
(n=20)\end{array}$ & $\begin{array}{l}\text { Olanzapine } \\
(n=\mid 5)\end{array}$ & $\begin{array}{l}\text { Total } \\
(n=5 I)\end{array}$ \\
\hline Males & 6 & 5 & 7 & $18(35 \%)$ \\
\hline Females & 10 & 15 & 8 & $33(65 \%)$ \\
\hline Mean age (years) & 41.5 & 42 & 41 & \\
\hline Delusional before treatment & 12 & 14 & 10 & $36(71 \%)$ \\
\hline Delusional after treatment & 3 & 4 & 2 & 9 \\
\hline Hallucinations before treatment & 10 & 5 & 3 & $18(35 \%)$ \\
\hline Hallucinations after treatment & 2 & 1 & 1 & 4 \\
\hline Suicidal behavior, prior treatment & 2 & 4 & 3 & $9(17 \%)$ \\
\hline History of other diagnoses (psychosis & 4 & 2 & 3 & $9(17 \%)$ \\
\hline \multicolumn{5}{|l|}{ NOS and schizoaffective disorder) } \\
\hline \multicolumn{5}{|l|}{ Medication } \\
\hline Citalopram & II & 16 & 10 & 37 \\
\hline Venlafaxine $X R$ & 5 & 4 & 5 & 14 \\
\hline Mean dose of novels (mg) & 1.7 & 278 & 11 & \\
\hline
\end{tabular}

Abbreviations: NOS, not otherwise specified; $X R$, extended release. 
Table 2 Paired t-test: Changes in the CGI-S, HAMD-2I, and BPRS scores, and weight at 8 weeks

\begin{tabular}{|c|c|c|c|c|c|c|}
\hline \multirow[t]{2}{*}{ Measure } & \multicolumn{2}{|c|}{$\begin{array}{l}\text { Risperidone } \\
(n=16) \\
\text { Df: } 15\end{array}$} & \multicolumn{2}{|c|}{$\begin{array}{l}\text { Quetiapine } \\
(n=20) \\
\text { Df: } 19\end{array}$} & \multicolumn{2}{|c|}{$\begin{array}{l}\text { Olanzapine } \\
(n=15) \\
\text { Df: I } 4\end{array}$} \\
\hline & $t$ value & $P$ & $t$ value & $P$ & $t$ value & $P$ \\
\hline CGI-S & 13.7 & 0.001 & 30.7 & 0.001 & 12.8 & 0.001 \\
\hline HAMD-2I & 9.7 & 0.001 & 17.3 & 0.001 & 14.2 & 0.001 \\
\hline BPRS & 13 & 0.001 & 20.7 & 0.001 & 11.3 & 0.001 \\
\hline Weight (lb) & -2.5 & 0.02 & -1.0 & 0.3 & 0.9 & 0.01 \\
\hline
\end{tabular}

Abbreviations: BPRS, Brief Psychiatric Rating Scale; CGI-S, Clinical Global Impression-Severity subscale; DF, degrees of freedom; HAMD-2I, The depression Hamilton Scale.

one female patient who exhibited galactorrhea. Among the olanzapine patients, one reported mild hand tremor. No extrapyramidal symptoms were reported among quetiapine patients. At the end of the trial, there was one patient switched from risperidone to quetiapine due to akathisia and another from olanzapine to quetiapine due to excessive sedation and poor adherence. There was slight weight gain with the three novels augmentation over the 8 weeks; however, this was statistically significant at 8 weeks in only the olanzapine group that exhibited a $4.2 \mathrm{lb}$ weight gain $(P>0.04)$ versus a $1.5 \mathrm{lb}$ weight gain $(P>0.7)$ in the risperidone group and a $1 \mathrm{lb}$ weight gain $(P>0.4)$ in the quetiapine group (Table 2).

\section{Discussion}

In the current open-label, naturalistic study, we compared adjunctive treatments using three atypical antipsychotics, in patients with psychotic depression. We found that the three novel antipsychotic agents quetiapine, olanzapine, and risperidone were equally effective in the treatment of major depressive episodes with psychotic features and that the three drugs were equally tolerated, without significant differences in reported side effects.

It is not surprising that atypical antipsychotics could relieve psychotic symptoms in depression and other mood disorders, since the evidence is strong for their efficacy in relieving psychotic symptoms in schizophrenia and schizoaffective disorders. Many studies have found that novel antipsychotics have an antidepressant spectrum of activity in addition to their antipsychotic effects, in patients with schizophrenia or schizoaffective disorders. ${ }^{23-28}$

While there is a plethora of literature to support the efficacy of the novel antipsychotics olanzapine, risperidone, and quetiapine in improving depressive symptoms in patients with schizophrenia, these results have been viewed as very preliminary by some investigators. ${ }^{29}$ However, analysis of variance did not show significant differences in weight changes between the three treatment groups (Table 3).

The novel antipsychotics have also been proven to have efficacy in relieving both manic and depressive symptoms in patients with mixed bipolar disorders, bipolar depression, and in refractory depressive states. Also, recently there has been growing evidence for the efficacy of the atypical antipsychotics, especially quetiapine fumarate $\mathrm{XR}$, as monotherapy in unipolar depression., ${ }^{9,10}$

More recently, quetiapine monotherapy has demonstrated efficacy in the acute phase of refractory major depressive disorder, with or without psychotic symptoms. For example, in a number of randomized, placebo-controlled

Table 3 Changes in the CGI-S, HAMD-2I, BPRS scores, and weight over 8 weeks

\begin{tabular}{|c|c|c|c|c|c|c|c|c|c|c|c|}
\hline \multirow[t]{3}{*}{ Measure } & \multicolumn{3}{|c|}{ Risperidone $(n=16)$} & \multicolumn{3}{|c|}{ Quetiapine $(n=20)$} & \multicolumn{3}{|c|}{ Olanzapine $(n=15)$} & \multirow[t]{3}{*}{$F$ value } & \multirow[t]{3}{*}{$P$} \\
\hline & \multirow[t]{2}{*}{$M(S D)$} & \multicolumn{2}{|l|}{$95 \% \mathrm{Cl}$} & \multirow[t]{2}{*}{$M(S D)$} & \multicolumn{2}{|l|}{$95 \% \mathrm{Cl}$} & \multirow[t]{2}{*}{$M(S D)$} & \multicolumn{2}{|l|}{$95 \% \mathrm{Cl}$} & & \\
\hline & & Upper & Lower & & Upper & Lower & & Upper & Lower & & \\
\hline \multicolumn{12}{|l|}{ CGI-S } \\
\hline Baseline & $4.4(0.50)$ & 4.7 & 4.2 & $4.7(0.72)$ & 5.1 & 4.4 & $5.1(0.74)$ & 5.5 & 4.7 & 4.2 & 0.02 \\
\hline 8 weeks & $2.3(0.50)$ & 2.6 & 2.2 & $2.5(0.61)$ & 2.8 & 2.2 & $2.7(0.62)$ & 3.0 & 2.3 & 1.5 & 0.24 \\
\hline \multicolumn{12}{|l|}{ HAMD-2I } \\
\hline Baseline & $31.2(4.4)$ & 33.5 & 28.9 & $34.0(6.1)$ & 36.7 & 31.1 & $31(6.6)$ & 35.1 & 27.7 & 1.2 & 0.30 \\
\hline 8 weeks & $18.6(4.8)$ & 21.1 & 16.0 & $17.8(4.9)$ & 20.1 & 15.5 & $18(5.0)$ & 20.7 & 15.0 & 0.12 & 0.90 \\
\hline \multicolumn{12}{|l|}{ BPRS } \\
\hline Baseline & $49.1(5.7)$ & 52.1 & 46.0 & $56.8(6.8)$ & 60.0 & 53.6 & $46.5(5.8)$ & 49.7 & 43.3 & 13.4 & 0.01 \\
\hline 8 weeks & $30.1(3.8)$ & 32.7 & 28.6 & $22.4(5.0)$ & 34.7 & 30.1 & $29.4(4.0)$ & 31.6 & 27.2 & 2.1 & 0.13 \\
\hline \multicolumn{12}{|l|}{ Weight (lb) } \\
\hline Baseline & $188(38)$ & 208 & 167 & 161 (44) & 182 & $14 \mid$ & $170(22)$ & 182 & 157 & 2.3 & 0.11 \\
\hline 8 weeks & $189(39)$ & 209 & 168 & $\mid 70(5 \mid)$ & 195 & 146 & $167(22)$ & 179 & 155 & 1.3 & 0.28 \\
\hline
\end{tabular}

Abbreviations: BPRS, Brief Psychiatric Rating Scale; CGI-S, Clinical Global Impression-Severity subscale; Cl, confidence interval; HAMD-2I, Hamilton Rating Scale for Depression-2I item; M, mean; SD, standard deviation. 
studies, the authors concluded that the use of the atypical antipsychotic agents olanzapine, risperidone, quetiapine, and ziprasidone as adjunctive treatment with antidepressants may be a viable option in treatment-resistant major depressive disorder. ${ }^{30-34}$ For example, in an open trial, Matthews et $\mathrm{al}^{30}$ examined the efficacy of olanzapine $5-20 \mathrm{mg}$ /day plus fluoxetine $20-80 \mathrm{mg} /$ day, in 27 patients with DSM-IVdefined major depressive disorder with psychotic features. The investigators found a favorable response rate $-66.7 \%$ for depression and $59.3 \%$ for psychosis. The authors concluded that the combination of olanzapine and fluoxetine appears to be a promising, safe, and effective treatment for psychotic depression. ${ }^{30}$

Also, previous studies have demonstrated efficacy and tolerance of augmenting tricyclic antidepressants with traditional antipsychotics in patients with psychotic depression. For example, in a 6 week, multicenter, doubleblind, parallel group trial, Muller-Siecheneder et $\mathrm{al}^{35}$ examined the efficacy and tolerance of risperidone versus haloperidol and amitriptyline for the treatment of patients with a combined psychotic and depressive syndrome, in a heterogenous group of psychotic patients, with major depressive symptoms. The results of this trial suggested that the therapeutic effect of haloperidol and amitriptyline augmentation was superior to risperidone, in the total group of patients with combined psychotic and depressive symptoms. ${ }^{35}$ However, more recent studies demonstrated robust efficacy and tolerability when risperidone was used to augment SSRI antidepressants for the acute treatment of patients suffering from both psychotic and nonpsychotic major depressive episodes..$^{36,37}$

It is well established that atypical antipsychotic agents are a safer and more effective alternative to the widespread practice of maintenance adjunctive treatment with traditional neuroleptic agents, in patients with mood disorder. Although longer-term data are becoming available, most of the available evidence has come from short-term and studies that were not controlled. Despite the lack of long-term studies in both psychotic and nonpsychotic depression, the novel agents can serve as useful adjunctive treatments with the standard antidepressants used in mood disorders, as their efficacy in bipolar disorder has been established. ${ }^{38}$ To date, no differences in efficacy between the different novels have been established. However, differences in the side effect of weight gain may be even more relevant in major depression than in schizophrenia, due to the need for standard antidepressants that often potentiate such weight gain.

\section{What are the possible mechanisms for novel antipsychotics' action as novel antidepressants?}

There is extensive evidence to implicate the dysregulation of noradrenergic, serotonergic, and dopaminergic neurotransmission in the pathophysiology of mood disorders. The receptor profile of the atypical antipsychotic agents olanzapine, risperidone, and quetiapine, and their demonstrated efficacy in schizophrenia, is also consistent with antidepressant efficacy, and this has been recently demonstrated..$^{39}$ In one study, it was hypothesized that in rats, the combined administration of novel antipsychotics with SSRI antidepressants, such as flouxetine, resulted in synergistic antidepressant effects, by increasing the dopamine and norepinephrine levels significantly in the rat prefrontal cortex. It is well known that clinically effective antipsychotics are potent 5HT-2a receptor antagonists. Olanzapine, for example has demonstrated a high occupancy of $5 \mathrm{HT} 2$ receptors.$^{40} \mathrm{~A}$ dopamine role in depression has been suggested and discussed in some length by others, but this has to be considered carefully because dopamine antagonists and receptor blockers can give rise to an animal model similar to depression with psychomotor retardation. ${ }^{41}$ Further laboratory research, as well as clinical controlled studies, is needed to examine and clarify the different mechanisms implicated in the antidepressant effects of the novel antipsychotic agents, which remain poorly understood.

\section{Limitations of the study}

Although, in the current study, data were prepared and gathered in a precise and comprehensive manner to minimize missing data, this study was essentially a naturalistic one. Data were collected in an open fashion, and randomization was not utilized to assign the atypical antipsychotic medication to patient groups. There was no control placebo group, and the overall sample size was small. It was difficult to interpret the weight changes because of the small patient sample and the unavailability of body mass index measures, and the metabolic risks were not examined.

\section{Conclusion}

In this study we examined and compared the efficacy of the three novel agents risperidone, quetiapine, and olanzapine in psychotic depressed patients, and the three novels showed equivalent efficacy and tolerability during the acute phases of the illness. Perhaps ongoing studies will prove in future that the atypical antipsychotic agents studied here can also act and 
will be tolerated singly or in combination with atypical antidepressants, at least in a subgroup of depressed patients.

However, both the risks and benefits of these agents should be taken into account for an individual patient with major depression. To examine the efficacy and tolerance of long-term administration in nonpsychotic patients, controlled and long-term studies are needed. It remains unclear as to how long adjunctive antipsychotic treatment should be administered to depressed patients with psychotic features. This is particularly important given the fact that there is high risk for side effects with long-term use of atypical antipsychotic medications. Future research may shed light on to this issue. The available data suggest that this may need to be tailored to each patient.

\section{Acknowledgment}

Author would like to thank Dr Claudio Violato for his kind editorial comments.

\section{Disclosure}

The author reports no conflicts of interest in this work.

\section{References}

1. Ohayon MM, Schatzberg AF. Prevalence of depressive episodes with psychotic features in general population. Am J psychiatry. 2002; 159(11):1855-1861.

2. Rothschild AJ, Winer J, Flint AJ, et al; Study of Pharmacotherapy of Psychotic Depression (STOP-PD) Collaborative Study Group. Missed diagnosis of psychotic depression at 4 academic medical centers. J Clin Psychiatry. 2008;69(8):1293-1296.

3. Schatzberg AF. New approaches to managing psychotic depression. J Clin Psychiatry. 2003;64 Suppl 1:19-23.

4. Rothschild AJ, Samson JA, Bessette MP, Carter-Campbell JT. Efficacy of the combination of fluoxetine and perphenazine in the treatment of psychotic depression. J Clin Psychiatry. 1993;54(9):338-342.

5. Wolfersdorf M, Barg T, König F, Leibfarth M, Grünewald I. Paroxetine as antidepressant in combined antidepressant-neuroleptic therapy in delusional depression: observation of clinical use. Pharmacopsychiatry. 1995;28(2):56-60.

6. Weizman R, Weizman A. Use of atypical antipsychotics in mood disorders. Curr Opin Investig Drugs. 2001;2(7):940-945.

7. Möller HJ. Antidepressive effects of traditional and second generation antipsychotics: a review of the clinical data. Eur Arch Psychiatry Clin Neurosci. 2005;255(2):83-93.

8. Leadholm AK, Rothschild AJ, Nolen WA, Bech P, Munk-Jørgensen P, Ostergaard SD. The treatment of psychotic depression: is there consensus among guidelines and psychiatrists? J Affect Disord. 2013;145(2): 214-220.

9. Maneeton N, Maneeton B, Srisurapanont M, Martin SD. Quetiapine monotherapy in acute phase for major depressive disorder: a metaanalysis of randomized, placebo-controlled trials. BMC Psychiatry. 2012;12:160.

10. Weisler RH, Montgomery SA, Earley WR, Szamosi J, Lazarus A. Efficacy of extended release quetiapine fumarate monotherapy in patients with major depressive disorder: a pooled analysis of two 6-week, double-blind, placebo-controlled studies. Int Clin Psychopharmacol. 2012;27(1):27-39.
11. Katila H, Mezhebovsky I, Mulroy A, et al. Randomized, double-blind study of the efficacy and tolerability of extended release quetiapine fumarate (quetiapine XR) monotherapy in elderly patients with major depressive disorder. Am J Geriatr Psychiatry. Epub June 7, 2012.

12. El-Khalili N, Joyce M, Atkinson S, et al. Extended-release quetiapine fumarate (quetiapine XR) as adjunctive therapy in major depressive disorder (MDD) in patients with an inadequate response to ongoing antidepressant treatment: a multicentre, randomized, double-blind, placebo-controlled study. Int J Neuropsychopharmacol. 2010;13(7): 917-932.

13. Sanford M, Keating GM. Quetiapine: a review of its use in the management of bipolar depression. CNS Drugs. 2012;26(5):435-460.

14. Thase ME, Demyttenaere K, Earley WR, Gustafsson U, Udd M, Eriksson H. Extended release quetiapine fumarate in major depressive disorder: analysis in patients with anxious depression. Depress Anxiety. 2012;29(7):574-586.

15. Sheehan DV, Locklear J, Svedsäter H, Datto C. Long-term functioning and sleep quality in patients with major depressive disorder treated with extended-release quetiapine fumarate. Int Clin Psychopharmacol. 2012;27(5):239-248.

16. Gabriel A. The extended-release formulation of quetiapine fumarate (quetiapine XR) adjunctive treatment in partially responsive generalized anxiety disorder (GAD): an open label naturalistic study. Clin Ter. 2011;162(2):113-118.

17. Sajatovic M, Mullen JA, Sweitzer DF. Efficacy of quetiapine and risperidone against depressive symptoms in outpatients with psychosis. J Clin Psychiatry. 2002;63(12):1156-1163.

18. American Psychiatric Association. Diagnostic and statistical manual of mental disorders, fourth edition, DSM-IV. Washington, DC: APA; 1994.

19. Sheehan DV, Lecrubier Y, Sheehan KH, et al. The Mini-International Neuropsychiatric Interview (M.I.N.I.): the development and validation of a structured diagnostic psychiatric interview for DSM-IV and ICD-10. J Clin Psychiatry. 1998;59 Suppl 20:22-33.

20. National Institute of Mental health. CGI: Clinical global impression. In: Guy W, Bonato RR, editors. Manual for the ECDEU Assessment Battery, 2nd ed. Bethesda, MD: US Department of Health, Education, and Welfare, National Institute of Mental Health; 1970.

21. Hamilton M. A rating scale for depression. J Neurol Neurosurg Psychiatry. 1960;23:56-62.

22. Overall JE, Gorham DR. The brief psychiatric rating scale. Psychol Rep. 1962;10:799-812.

23. Tollefson GD, Sanger TM, Lu Y, Thieme ME. Depressive signs and symptoms in schizophrenia: a prospective blinded trial of olanzapine and haloperidol. Arch Gen Psychiatry.1998;55(3):250-258.

24. Keck PE Jr, Strakowski SM, McElroy SL. The efficacy of atypical antipsychotics in the treatment of depressive symptoms, hostility, and suicidality in patients with schizophrenia. J Clin Psychiatry. 2000; 61 Suppl 3:4-9.

25. Emsley RA, Buckley P, Jones AM, Greenwood MR. Differential effect of quetiapine on depressive symptoms in patients with partially responsive schizophrenia. J Psychopharmacol. 2003;17(2):210-215.

26. Cheer SM, Wagstaff AJ. Quetiapine. A review of its use in the management of schizophrenia. CNS Drugs. 2004;18(3):173-199.

27. Levinson DF, Umapathy C, Musthaq M. Treatment of schizoaffective disorder and schizophrenia with mood symptoms. Am J Psychiatry. 1999;156(8):1138-1148.

28. Khouzam HR. Treatment of depressive mood in schizophrenia with the atypical antipsychotic quetiapine. Depress Anxiety. 2000;11(2): $80-82$.

29. Babinkostova Z, Stefanovski B. Forms of antipsychotic therapy: improved individual outcomes under personalised treatment of schizophrenia focused on depression. EPMA J. 2011;2(4):391-402.

30. Matthews JD, Bottonari KA, Polania LM, et al. An open study of olanzapine and fluoxetine for psychotic major depressive disorder: interim analyses. J Clin Psychiatry. 2002;63(12):1164-1170. 
31. Bhana N, Perry CM. Olanzapine: a review of its use in the treatment of bipolar I disorder. CNS Drugs. 2001; 15(11):871-904.

32. Barbee JG, Conrad EJ, Jamhour NJ. The effectiveness of olanzapine, risperidone, quetiapine, and ziprasidone as augmentation agents in treatment-resistant major depressive disorder. J Clin Psychiatry. 2004;65(7):975-981.

33. Papakostas GI, Shelton RC. Use of atypical antipsychotics for treatment-resistant major depressive disorder. Curr Psychiatry Rep. 2008;10(6):481-486.

34. Ravindran AV, Bradbury C, McKay M, da Silva TL. Novel uses for risperidone: focus on depressive, anxiety and behavioral disorders. Expert Opin Pharmacother. 2007;8(11):1693-1710.

35. Müller-Siecheneder F, Müller MJ, Hillert A, Szegedi A, Wetzel H, Benkert O. Risperidone versus haloperidol and amitriptyline in the treatment of patients with a combined psychotic and depressive syndrome. J Clin Psychopharmacol. 1998;18(2):111-120.
36. Ostroff RB, Nelson JC. Risperisone augmentation of selective serotonin reuptake inhibitors in major depression. J Clin Psychiatry. 1999;60(4): 256-259.

37. Viner MW, Chen Y, Bakshi I, Kamper P. Low dose risperidone augmentation of antidepressants in nonpsychotic depressive disorders with suicidal ideation. J Clin Psychopharmacol. 2003;23(1):104-106.

38. McIntyre R, Katzman M. The role of atypical antipsychotics in bipolar depression and anxiety disorders. Bipolar Disord. 2003;5 Suppl 2: $20-35$.

39. Myers JE, Thase ME. Risperidone: review of its therapeutic utility in depression. Psychopharmacol Bull. 2001;35(4):109-129.

40. Nyberg S, Farde L, Halldin C. A PET study of 5-HT2 and D2 dopamine receptor occupancy induced by olanzapine in healthy subjects. Neuropsychopharmacology. 1997;16(1):1-7.

41. Parker G, Malhi G. Are atypical antipsychotic drugs also atypical antidepressants? Aust N Z J Psychiatry. 2001;35(5):631-638.

\section{Publish your work in this journal}

Neuropsychiatric Disease and Treatment is an international, peerreviewed journal of clinical therapeutics and pharmacology focusing on concise rapid reporting of clinical or pre-clinical studies on a range of neuropsychiatric and neurological disorders. This journal is indexed on PubMed Central, the 'PsycINFO' database and CAS.
The manuscript management system is completely online and includes a very quick and fair peer-review system, which is all easy to use. Visit http://www.dovepress.com/testimonials.php to read real quotes from published authors.

\footnotetext{
Submit your manuscript here: http://www.dovepress.com/neuropsychiatric-disease-and-treatment-journal
} 